Louisiana State University

LSU Digital Commons

Faculty Publications

Department of Physics \& Astronomy

$1-1-2006$

\title{
The ligo gravitational wave observatories: Recent results and future plans
}

\author{
G. M. Harry \\ Massachusetts Institute of Technology \\ R. Adhikari \\ Massachusetts Institute of Technology \\ S. Ballmer \\ Massachusetts Institute of Technology \\ K. Bayer \\ Massachusetts Institute of Technology \\ J. Betzwieser \\ Massachusetts Institute of Technology
}

See next page for additional authors

Follow this and additional works at: https://digitalcommons.Isu.edu/physics_astronomy_pubs

\section{Recommended Citation}

Harry, G., Adhikari, R., Ballmer, S., Bayer, K., Betzwieser, J., Bochner, B., Burgess, R., Cadonati, L., Chatterji, S., Corbitt, T., Csatorday, P., Fritschel, P., Goda, K., Hefetz, Y., Katsavounidis, E., Lawrence, R., Macinnis, M., Marin, A., Mason, K., Mavalvala, N., Mittleman, R., Ottaway, D., Pratt, M., Regimbau, T., Richman, S., Rollins, J., Shoemaker, D., Smith, M., Van Putten, M., Weiss, R., Aulbert, C., Berukoff, S., \& Cutler, C. (2006). The ligo gravitational wave observatories: Recent results and future plans. The Tenth Marcel Grossmann Meeting: On Recent Developments in Theoretical and Experimental General Relativity, Gravitation and Relativistic Field Theories, 1, 308-336. https://doi.org/10.1142/9789812704030_0023

This Article is brought to you for free and open access by the Department of Physics \& Astronomy at LSU Digital Commons. It has been accepted for inclusion in Faculty Publications by an authorized administrator of LSU Digital Commons. For more information, please contact ir@lsu.edu. 


\section{Authors}

G. M. Harry, R. Adhikari, S. Ballmer, K. Bayer, J. Betzwieser, B. Bochner, R. Burgess, L. Cadonati, S. Chatterji, T. Corbitt, P. Csatorday, P. Fritschel, K. Goda, Y. Hefetz, E. Katsavounidis, R. Lawrence, M. Macinnis, A.

Marin, K. Mason, N. Mavalvala, R. Mittleman, D. J. Ottaway, M. Pratt, T. Regimbau, S. Richman, J. Rollins, D. H. Shoemaker, M. Smith, M. Van Putten, R. Weiss, C. Aulbert, S. J. Berukoff, and C. Cutler 


\section{THE LIGO GRAVITATIONAL WAVE OBSERVATORIES: RECENT RESULTS AND FUTURE PLANS}

G. M. HARRY, R. ADHIKARI, S. BALLMER, K. BAYER, J. BETZWIESER, B. BOCHNER, R. BURGESS, L. CADONATI, S. CHATTERJI, T. CORBITT, P. CSATORDAY, P. FRITSCHEL, K. GODA, Y. HEFETZ, E. KATSAVOUNIDIS,

R. LAWRENCE, M. MACINNIS, A. MARIN, K. MASON, N. MAVALVALA, R. MITTLEMAN, D. J. OTTAWAY, M. PRATT, T. REGIMBAU, S. RICHMAN,

J. ROLLINS, D. H. SHOEMAKER, M. SMITH, M. VAN PUTTEN, R. WEISS

LIGO - Massachusetts Institute of Technology, Cambridge, MA 02139, USA

C. AUlBERT, S. J. BERUKOFF, C. CUTLER, S. GRUNEWALD, Y. ITOH, B. KRISHNAN, B. MACHENSCHALK, S. MOHANTY, S. MUKHERJEE, H. NAUNDORF, M. A. PAPA, B. F. SCHUTZ, A. M. SINTES, P. R. WILLIAMS Albert-Einstein-Institut, Max-Planck-Institut für Gravitationsphysik, D-14476 Golm, Germany

C. COLACINO, K. DANZMANN, A. FREISE, H. GROTE, G. HEINZEL, K. KAWABE, P. KLOEVEKORN, H. LÜCK,K. MOSSAVI, S. NAGANO, A. RÜDIGER,

R. SCHILliNG, J. R. SMITH, A. WEIDNER, B. WILLKE, W. WINKLER

Albert-Einstein-Institut, Max-Planck-Institut für Gravitationsphysik, D-30167 Hannover, Germany

B. J. CUSACK, D. E. MCCLELLAND, S. M. SCOTT, A. C. SEARLE Australian National University, Canberra, 0200, Australia

R. W. P. DREVER, M. TINTO, R. WILLIAMS

California Institute of Technology, Pasadena, CA 91125, USA

A. BUONANNO, Y. CHEN, K. S. THORNE, M. VALLISNERI Caltech-CaRT, Pasadena, CA 91125, USA

B. ABBOtT, S. B. ANDERSON, M. ARAYA, H. ARMANDUlA, F. ASIRI, B. C. BARISH, M. BARNES, M. A. BARTON, B. BHAWAL,G. BILLINGSLEY, E. BLACK, K. BLACKBURN, L. BOGUE, R. BORK, D. BUSBY, L. CARDENAS, A. CHANDLER, J. CHAPSKY, P. CHARLTON, D. COYNE, T. D. CREIGHTON, E. D'AMBROSIO, R. DESALVO, H. DING, J. EDLUND, P. EHRENS, T. ETZEL,

M. EVANS, D. FARNHAM, M. FINE, A. GILLESPIE, D. GRIMMETT,

A. HARTUNIAN, J. HEEFNER, P. HOANG, M. HRYNEVYCH, A. IVANOV, L. JONES, D. JUNGWIRTH, W. KELLS, C. KING, P. KING, D. KOZAK,

A. LAZZARINI, M. LEI, K. LIBBRECHT, P. LINDQUIST, S. LIU, J. LOGAN,

T. T. LYONS, M. MAGESWARAN, K. MAILAND, W. MAJID, F. MANN, S. MÁRKA, E. MAROS, J. MASON, S. MESHKOV, O. MIYAKAWA, S. MIYOKI, B. MOURS, F. NOCERA, D. OUIMETTE, M. PEDRAZA, S. R. RAO, D. REDDING,

M. W. REGEHR, K. T. REILLY, K. REITHMAIER, L. ROBISON, J. ROMIE,

D. ROSE, P. RUSSELL, I. SALZMAN, G. H. SANDERS, V. SANNIBALE,

V. SCHMIDT, B. SEARS, S. SEEL, P. SHAWHAN, L. SIEVERS, M. R. SMITH, R. SPERO, M. C. SUMNER, J. SYLVESTRE, A. TAKAMORI, H. TARIQ, R. TAYLOR, S. TILAV, C. TORRIE, W. TYLER, S. VASS, L. WALLACE, B. WARE,

plenary: submitted to World Scientific on January 16, 2004 
D. WEBBER, A. WEINSTEIN, L. WEN, S. E. WHITCOMB, P. A. WILLEMS,

A. WILSON, H. YAMAMOTO, L. ZHANG, J. ZWEIZIG

LIGO - California Institute of Technology, Pasadena, CA 91125, USA

K. S. GANEZER

California State University Dominguez Hills, Carson, CA 90747, USA

S. BABAK, R. BALASUBRAMANIAN, D. CHURCHES, R. DAVIES, B. SATHYAPRAKASH, I. TAYLOR

Cardiff University, Cardiff, CF2 3YB, United Kingdom

N. CHRISTENSEN, C. EBELING

Carleton College, Northfield, MN 5505\%, USA

É. FLANAGAN

Cornell University, Ithaca, $N Y$ 14853, USA

T. NASH

Fermi National Accelerator Laboratory, Batavia, IL 60510, USA

S. PENN

Hobart and William Smith Colleges, Geneva, NY 14456, USA

S. DHURANDAR, R. NAYAK, A. S. SENGUPTA

Inter-University Centre for Astronomy and Astrophysics, Pune - 411007, India

D. BARKER, C. BARKER-PATTON, B. BLAND-WEAVER, D. COOK, C. GRAY,

M. GUENTHER, N. HINDMAN, M. LANDRY, M. LUBINSKI, O. MATHERNY,

L. MATONE, R. MCCARTHY, G. MENDELL, G. MORENO, J. MYERS,

V. PARAMESWARIAH, F. RAAB, H. RADKINS, K. RYAN, R. SAVAGE, P. SCHWINBERG, D. SIGG, C. VORVICK, J. WORDEN

LIGO Hanford Observatory, Richland, WA 99352, USA

R. ABBOTT, K. CARTER, M. COLES, T. EVANS, V. FROLOV, M. FYFFE,

A. M. GRETARSSON, M. HAMMOND, J. HANSON, J. KERN, A. KHAN,

J. KOVALIK, J. LANGDALE, M. LORMAND, B. O'REILLY, H. OVERMIER,

C. PARAMESWARIAH, R. RIESEN, A. RIZZI, S. RODDY, A. SIBLEY, G. STAPFER,

G. TRAYLOR, K. WATTS, R. WOOLEY, I. YAKUSHIN, M. ZUCKER

LIGO Livingston Observatory, Livingston, LA 70754, USA

V. CHICKARMANE, E. DAW, J. A. GIAIME, G. GONZÁLEZ, W. O. HAMILTON, 
W. W. JOHNSON, S. WEN

Louisiana State University, Baton Rouge, LA 70803, USA

N. ZOTOV

Louisiana Tech University, Ruston, LA 71272, USA

M. MCHUGH, J. T. WHELAN

Loyola University, New Orleans, LA 70118, USA

H. WALTHER

Max Planck Institut für Quantenoptik, D-85748, Garching, Germany

A. AGEEV, I. A. BILENKO, V. B. BRAGINSKY, V. P. MitROFANOV, K. V. TOKMAKOV, S. P. VYACHANIN

Moscow State University, Moscow, 119992, Russia

J. B. CAMP

NASA/Goddard Space Flight Center, Greenbelt, MD 207r1, USA

S. KAWAMURA

National Astronomical Observatory of Japan, Tokyo 181-8588, Japan

K. BELCZYNSKI, P. GRANDCLÉMENT, V. KALOGERA, C. KIM, P. NUTZMAN

Northwestern University, Evanston, IL 60208, USA

T. OLSON

Salish Kootenai College, Pablo, MT 59855, USA

S. YOSHIDA

Southeastern Louisiana University, Hammond, LA 70402, USA

R. BEAUSOleil, A. BUllington, R. L. BYER, D. DEBRA, M. M. FEJER, E. GUSTAFSON, C. HARDHAM, M. HENNESSY, W. HUA, B. LANTZ, N. A. ROBERTSON

Stanford University, Stanford, CA 94305, USA

P. R. SAULSON

Syracuse University, Syracuse, NY 13244, USA

L. S. FINN, N. HEPLER, B. J. OWEN, E. ROTTHOFF, K. SCHLAUFMAN, C. A. SHAPIRO, A. STUVER, T. SUMMERSCALES, P. J. SUTTON, M. TIBBitS, B. J. WINJUM

The Pennsylvania State University, University Park, PA 16802, USA

W. G. ANDERSON, M. DÍAZ, W. JOHNSTON, J. D. ROMANO, C. TORRES

The University of Texas at Brownsville and Texas Southmost College, Brownsville, TX

plenary: submitted to World Scientific on January 16, 2004 
78520, USA

D. UGOLINI

Trinity University, San Antonio, TX 78212, USA

P. AUFMUTH, S. BROZEK, C. FALLNICH, S. GOßLER, I. S. HENG, M. HEURS, K. KÖTTER, V. LEONHARDT, M. MALEC, V. QUETSCHKE, M. SCHREMPEL, S. TRAEGER, U. WEILAND, H. WELLING, I. ZAWISCHA

Universität Hannover, D-30167 Hannover, Germany

R. INGLEY, C. MESSENGER, A. VECCHIO

University of Birmingham, Birmingham, B15 2TT, United Kingdom

R. AMIN, J. CASTIGLIONE, R. COLDWELL, T. DELKER, S. KLIMENKO, G. MITSELMAKHER, G. MUELLER, M. RAKHMANOV, D. H. REITZE, H. RONG, A. SAZONOV, Q. Z. SHU, D. B. TANNER, B. F. WHITING, S. WISE University of Florida, Gainsville, FL 32611, USA

B. BARR, R. BENNETt, G. CAGNOli, C. A. CANTley, M. M. CASEY, D. R. M. CROOKS, R. J. DUPUIS, E. J. ELLIFFE, A. GRANT, A. HEPTONSTALL, M. HEWITSON, J. HOUGH, O. JENNRICH, S. KILLBOURN,

C. J. KILLOW,P. MCNAMARA, G. NEWTON, M. PITKIN, M. PLISSI, D. I. ROBERTSON, S. ROWAN, K. SKELDON, P. SNEDDON, K. A. STRAIN, H. WARD, G. WOAN

University of Glasgow, Glasgow, G12 8QQ, United Kingdom

D. CHIN, R. GUSTAFSON, K. RILES

University of Michigan, Ann Arbor, MI 48109, USA

J. E. BRAU, R. FREY, M. ITO, I. LEONOR, R. RAHKOLA, R. SCHOFIELD, D. STROM

University of Oregon, Eugene, OR 97403, USA

W. E. BUTLER

University of Rochester, Rochester, NY 14627, USA

B. Allen, P. R. BRADY, D. A. BROWN, J. D. E. CREIGHTON, S. KORANDA, A. OTTEWILL, X. SIEMENS, A. G. WISEMAN

University of Wisconsin-Milwaukee, Milwaukee, WI 53201, USA

S. BOSE

Washington State University, Pullman, WA 99164, USA

The LIGO Scientific Collaboration, http://www.ligo.org

\footnotetext{
The LIGO interferometers are operating as gravitational wave observatories, with a noise level near an order of magnitude of the goal and the first scientific data recently taken. This data has been analyzed for four different categories of gravitational wave sources; millisecond bursts, inspiralling binary neutron stars, periodic waves from a known pulsar, and stochastic background. Research and development is also underway for the next generation LIGO detector, Advanced LIGO.
}

plenary: submitted to World Scientific on January 16, 2004 


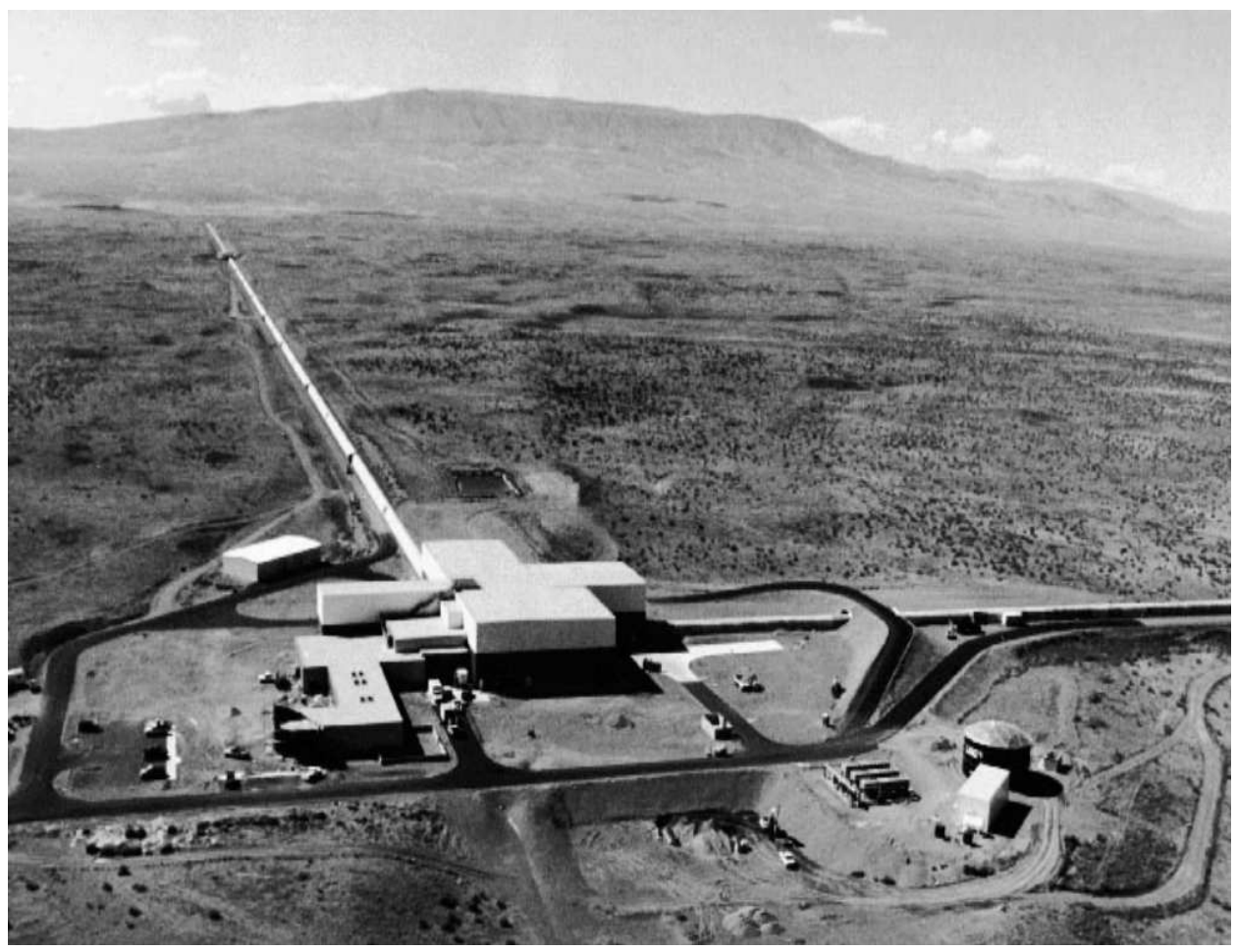

Figure 1. The LIGO Hanford Observatory

\section{Introduction}

The LIGO (Laser Interferometer Gravitational Wave Observatory) laboratory has constructed gravitational wave observatories at two sites in the continental United States; one near Richland, Washington on the Department of Energy Hanford Reservation (shown in Fig. 1), and the second near Baton Rouge, Louisiana in Livingston Parish (shown in Fig. 2). They are separated by $3000 \mathrm{~km}$ or about $10 \mathrm{~ms}$ of light travel time. Construction of the facilities began in 1994. These observatories consist of one (at Livingston) or two (at Hanford) laser interferometers designed to have high sensitivity to differential length changes in two perpendicular arms. The Livingston interferometer and one of the Hanford interferometers are $4 \mathrm{~km}$ in length, while the other Hanford interferometer is $2 \mathrm{~km}$ in length. The two observatories are as close to aligned as the curvature of the Earth will allow.

The tensor gravitational waves emitted by astronomical sources will cause a change in differential length between two perpendicular directions ${ }^{1}$. This strain, however, is expected to be extremely small, on the order of $10^{-21}$ in the acoustic frequency band that LIGO is sensitive to. The LIGO interferometers have been carefully designed so that they may be sensitive to small differential displacements. The interferometer measures the position of masses at the ends of each arm, which

plenary: submitted to World Scientific on January 16, 2004 


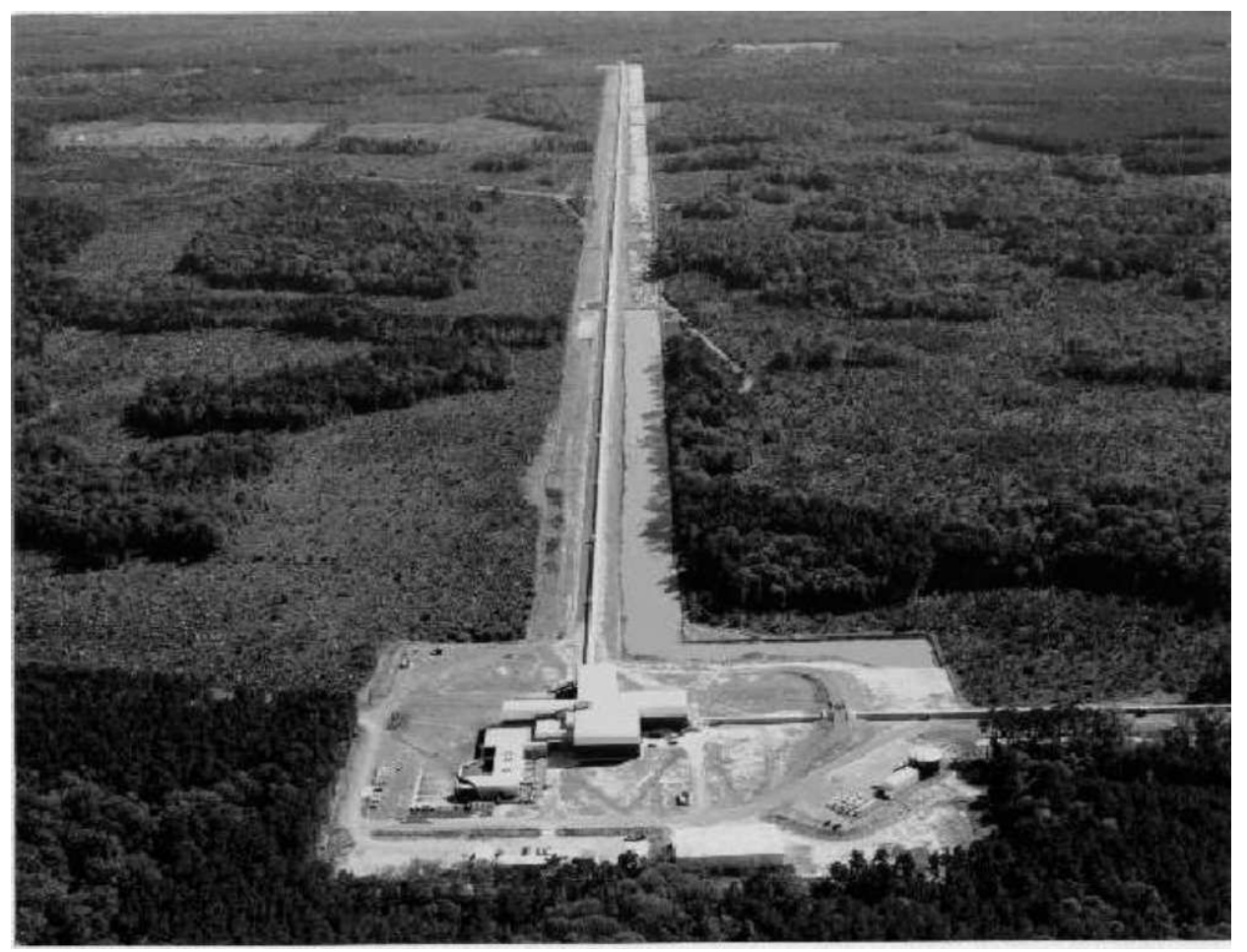

Figure 2. The LIGO Livingston Observatory

are held fixed in the vertical direction, but are nearly free along the optical path of the laser. This is accomplished by hanging the masses as pendulums using wire slings. This freedom of motion allows the masses to respond to the changing spacetime created by the wave without interference from terrestrial forces. The arms of each detector are kilometers long, so that the strain of the wave will create a relatively large change in displacement. This displacement is read out using a Michelson interferometer, so each mass is a mirror which allows the 1 micron laser light to reflect off of it. The gravitational wave signal increases with the interaction time with the light, so each arm includes a Fabry-Perot cavity made up of the end test mass and input test mass near the beamsplitter. The output port of the interferometer is held at a dark fringe. To further increase the signal, additional power buildup is accomplished by placing another mirror at the bright port. The entire optical path of the laser, including the kilometer long arms, must be held in a vacuum of below $10^{-8}$ mbar. The optical layout is shown in Figure 3.

The sensitivity is ultimately limited by fundamental noise sources inherent in the design. At the highest frequencies, above about $200 \mathrm{~Hz}$, the sensitivity is limited by shot noise from the laser. This is reduced by increasing the laser power, as the signal rises faster with power than the noise. At intermediate frequencies, between about $40 \mathrm{~Hz}$ and $200 \mathrm{~Hz}$, noise from thermally activated motion of the suspensions

plenary: submitted to World Scientific on January 16, 2004 


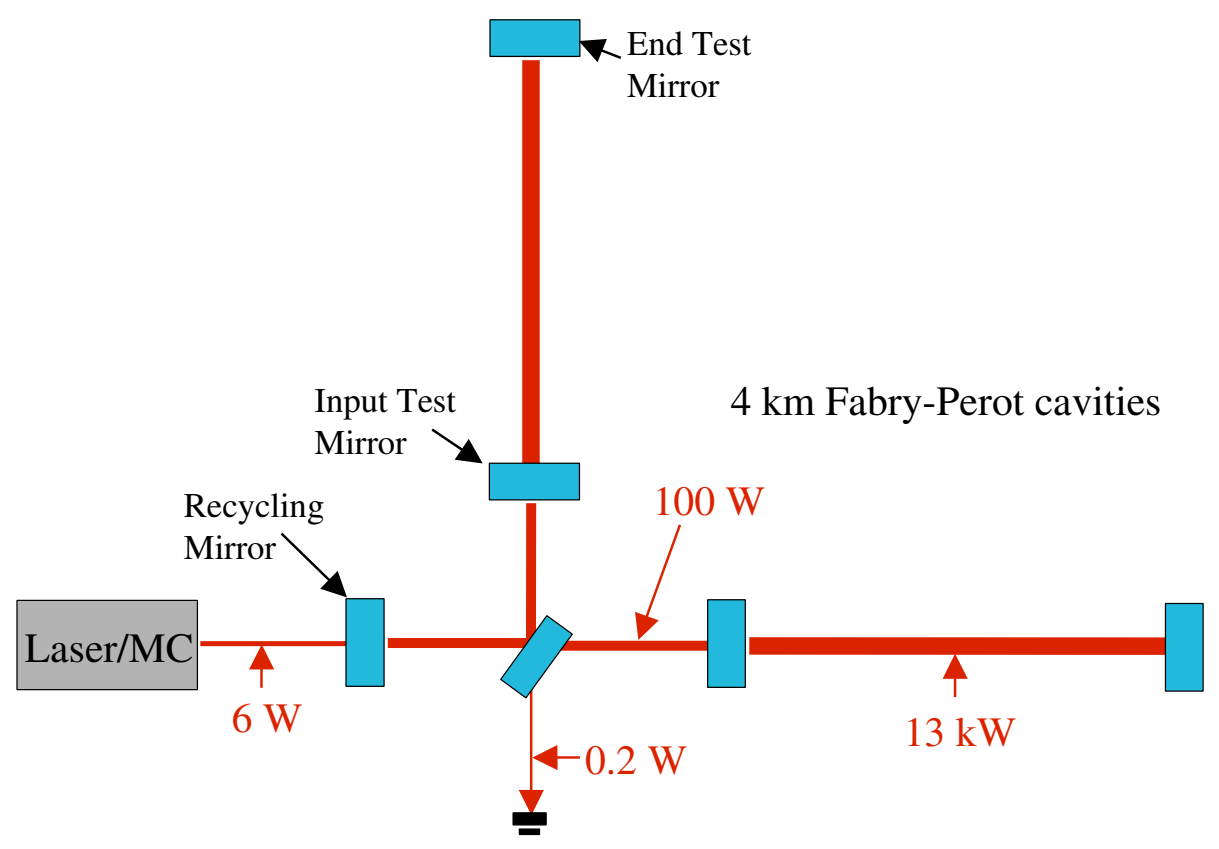

Figure 3. The optical layout of a LIGO interferometer showing the laser power at various points. A Michelson interferometer is supplemented with Fabry-Perot arm cavities $4 \mathrm{~km} \mathrm{long}$, and a power recycling mirror to reflect light back into the system as it leaves at the bright port.

dominate the noise budget. This noise is controlled by careful choice of materials and processes for the test masses, suspension wires, and nearby hardware. At low frequencies, below $40 \mathrm{~Hz}$, seismic noise dominates. The coupling between the inherent motion of the ground and the mirror's motion is reduced by placing the test mass suspension on vibration isolation stacks. The sensitivity goal for the current $4 \mathrm{~km}$ long interferometers is shown in Figure 4. This goal reaches a noise of about $2 \times 10^{-23}$ strain $/ \sqrt{\mathrm{Hz}}$ at $150 \mathrm{~Hz}$. More details about the LIGO interferometers is given below in Section 2. A more complete description of the LIGO detectors is available in $\operatorname{Ref}^{2}$.

The first operation of a LIGO interferometer occurred in October of 2000. Since then, the interferometers have been in a commissioning phase, where work to reduce noise and improve performance has alternated with periods of running the interferometer to assess its status. A history of the noise in the Livingston interferometer is shown in Figure 5. These periods of operation, called engineering runs, were also used to collect data to test data handling, archiving, and the analysis software that was being developed in parallel with the instruments.

In the summer of 2002, the sensitivity had reached a level where data collected from a short period of operating could be expected to achieve limits on observable gravitational waves that would be comparable to or even better than previous experimental limits. This data collection run, referred to as the first LIGO Science

plenary: submitted to World Scientific on January 16, 2004 


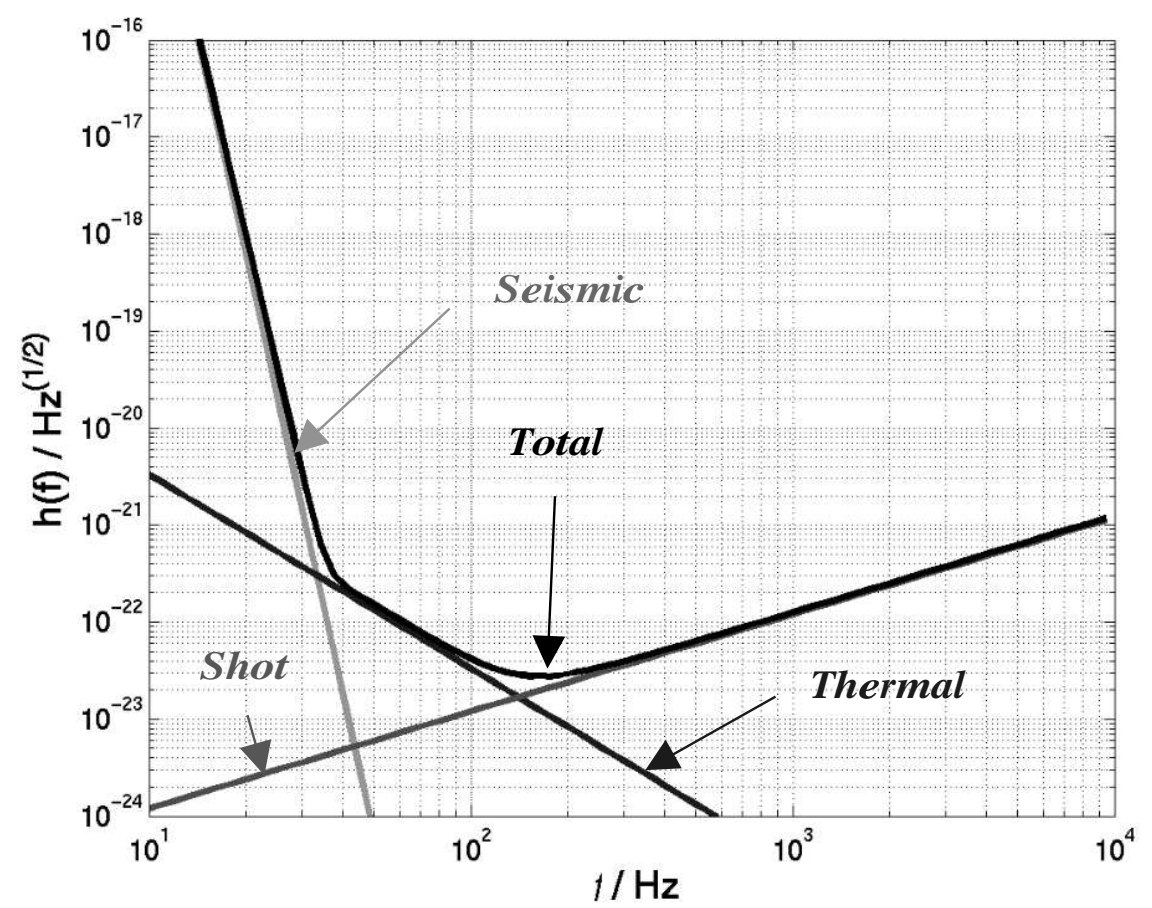

Figure 4. The sensitivity goal for the $4 \mathrm{~km}$ LIGO interferometers. Low frequency sensitivity is limited by seismic noise, the middle frequency band by thermal noise from both the suspension and test mass, and high frequencies by shot noise.

Run (S1), occurred between August 23 and September 9, 2002. GEO $600^{3}$ also took data during this time period, to allow for coordinated data analysis. The data from all interferometers was analyzed for four different categories of gravitational waves; burst events, inspirals of binary neutron stars, continuous waves coming from a gravitational pulsar, and a stochastic background. Results of these analyses are given below in Section 3.

Simultaneous with commissioning and data taking, research and development has been going on throughout the LIGO Scientific Collaboration on technologies for the next generation interferometers. In 1998, a preliminary design was put forth for the Advanced LIGO interferometer, which incorporates technology advances made since the initial LIGO interferometers were constructed. Key among these technologies are a higher power laser, larger test masses made of single crystal sapphire, fused silica ribbon suspensions, and an additional mirror placed at the output port. Research for Advanced LIGO reached a mature enough state that a construction proposal was submitted to the US National Science Foundation in February of 2003. Details of Advanced LIGO are discussed below in Section 4.

plenary: submitted to World Scientific on January 16, 2004 


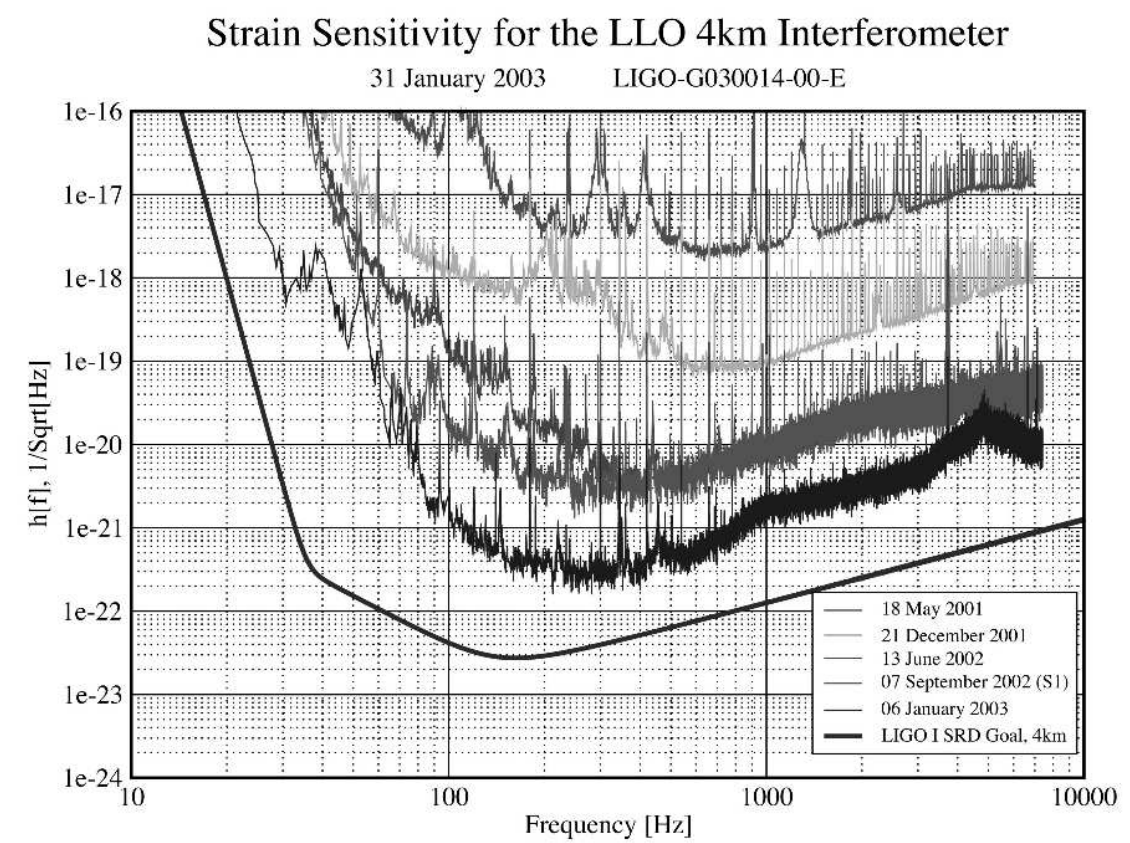

Figure 5. Noise in the LIGO Livingston interferometer through time.

\section{The LIGO Interferometers}

\subsection{Laser and Input Optics}

Each interferometer is fitted with a continuous-wave, Nd:YAG laser operating in the $\mathrm{TEM}_{00}$ Gaussian spatial mode at $1064 \mathrm{~nm}$, and capable of delivering $10 \mathrm{~W}$ of power $^{4}$. A small amount of the light coming out of the laser is sent to a reference cavity to stabilize the frequency to at least $0.1 \mathrm{~Hz} / \sqrt{\mathrm{Hz}}$ within the gravitational wave band. The remainder of the light goes through a triangular pre-mode cleaner to strip off light in modes other than $\mathrm{TEM}_{00}$. This pre-mode cleaner also provides passive filtering of intensity noise. During the S1 run, intensity noise was around $10^{-7} / \sqrt{\mathrm{Hz}}$. However, the intensity noise control system was only partially implemented, it is expected to be able to deliver intensity noise as low as $10^{-8} / \sqrt{\mathrm{Hz}}$.

The light from the laser then passes into the vacuum and the input optics subsystem. During the S1 run, only $1 \mathrm{~W}$ of laser light entered the input optics in the Livingston and Hanford $4 \mathrm{~km}$ interferometers, and about $6 \mathrm{~W}$ for the Hanford $2 \mathrm{~km}$ interferometer. The light first passed through a mode cleaner, three suspended optics forming a triangular cavity $24 \mathrm{~m}$ in length with a finesse of about 1350 . The mode cleaner stabilizes the beam size, position, and pointing of the laser light. It also acts as an additional frequency reference. Feedback to the laser from the mode

plenary: submitted to World Scientific on January 16, 2004 
cleaner reduced the frequency noise to around $10^{-3} \mathrm{~Hz} / \sqrt{\mathrm{Hz}}$ during the S1 run. After the mode cleaner is a Faraday isolator, which diverts light returning from the interferometer away from the laser and into a photodetector. Finally, the light goes through a mode-matching telescope made of three suspended optics. This telescope increases the beam size to about $4 \mathrm{~cm}\left(1 / \mathrm{e}^{2}\right.$ radius in power) to match the mode of the arm cavities.

\subsection{Interferometer Optics}

The optics that form the main part of the interferometer ${ }^{5,6}$ are made from fused silica glass. The end mirrors and input mirrors that define the Fabry-Perot cavities and the recycling mirror are $25 \mathrm{~cm}$ in diameter and $10 \mathrm{~cm}$ thick, while the beamsplitter is the same diameter by only $4 \mathrm{~cm}$ thick. The radii of curvature for the cavity optics are designed so the arm cavities have a stability factor

$$
g=\left(1-L / R_{1}\right)\left(1-L / R_{2}\right)
$$

of 0.33 for the $4 \mathrm{~km}$ long arms and 0.67 for the $2 \mathrm{~km}$ long arms. The surface figure of the polished optics is better than $1 \mathrm{~nm}$.

The coatings on the mirrors are alternating layers of silica and tantala. The optical absorption is less than 1 part-per-million (ppm), and the scatter is less than $70 \mathrm{ppm}$. The transmission of the end mirrors is chosen to be as low as practical, about $12 \mathrm{ppm}$. The input mirrors have transmissions of $2.7 \%$, which results in a pole frequency for the Fabry-Perot cavities of $85 \mathrm{~Hz}$ for the $4 \mathrm{~km}$ long arms and $170 \mathrm{~Hz}$ for the $2 \mathrm{~km}$ long arms. The beamsplitter transmission is $50 \pm 0.5 \%$. The recycling mirror has a $2.7 \%$ transmission, resulting in a recycling gain of about 50 .

The absorption of optical power in the mirror substrates and coatings causes significant heating and thermal lensing of the optics. Thus, the radii of curvature specification assumed a level of thermal lensing. The low light level used during the $\mathrm{S} 1$ run caused reduced heating and thermal lensing in the $4 \mathrm{~km}$ interferometers. In addition, the Hanford $2 \mathrm{~km}$ interferometer had an anti-reflection coating for one of the input mirrors that did not meet the specification. These issues resulted in lower recycling gain for all interferometers during S1 than the design goal.

The silica substrates for the mirrors were chosen, in part, for their low mechanical loss. Through the fluctuation-dissipation theorem, this low loss results in low, off-resonance thermal noise. The mirror geometry assures that the lowest internal mode of the mirrors is above the gravitational wave band, at about $6700 \mathrm{~Hz}$. The coating can also cause significant thermal noise ${ }^{7}$, however for the particular materials ${ }^{8}$ and beam radii used here, coating thermal noise is not expected to be a limiting noise source. Mechanical Q's of in situ mirrors have been measured to check this expectation. The addition of glued-on magnets and suspension attachments (see below) can degrade some modal Q's, but because of their distance from the reflected laser, will have a negligible effect on thermal noise. Modal Q's are found to be between $2 \times 10^{5}$ and $1.6 \times 10^{7}$, and finite element modeling predicts that the thermal noise will be near the design goal, and well below the limiting noise during the S1 run.

plenary: submitted to World Scientific on January 16, 2004 


\subsection{Seismic Isolation and Suspensions}

To reduce the effect of ground motion on the optics, the optics are supported from a vibration isolation stack; an alternating series of masses and springs. This stack is composed of four layers of passive isolation ${ }^{9}$. The final stage is the optical table that holds the suspension. The internal modes of this isolation structure are between 1.5 and $12 \mathrm{~Hz}$, with Q's typically between 10 and 30. The transfer functions between motion on the ground and motion at the optical table begins to roll off as $f^{-8}$ above the highest frequency mode, reaching an isolation factor of about $10^{6}$ at $50 \mathrm{~Hz}$.

Each interferometer optic is suspended from a steel piano wire pendulum. A schematic drawing is shown in Fig. 6. The single loop of wire is loaded to near $40 \%$ of its yield strength. Small glass rods with a notch in them to hold the wire are glued onto the optic on each side a few millimeters above the center of mass. This helps define a clear break-off point for the wire, and reduces friction. This results in suspension modes of $0.74 \mathrm{~Hz}$ for the pendulum mode, 0.5 for yaw, $0.6 \mathrm{~Hz}$ for pitch, $12 \mathrm{~Hz}$ for the vertical bounce mode, $18 \mathrm{~Hz}$ for the roll mode, and $345 \mathrm{~Hz}$ (and multiples thereof) for the violin modes. All of these modes, except the first few violin modes, are outside of the gravitational wave band. Thermal noise from the violin modes is visible in the detection band in a very narrow region around the resonance. Off-resonance thermal noise is minimized by using the stressed steel wire and careful assembly of the suspension. Suspension thermal noise is expected to be a limiting noise source at the LIGO design goal (see Fig. 4). Measured Q's on the low frequency pendulum modes of typically 2 to $410^{5}$ show that suspension thermal noise is below the sensitivity of the S1 run.

The necessary control forces for the interferometer optics are applied using the suspension system. Magnets made from Nd:Fe:B, 4 on the back and 1 one the side, are epoxied onto each optic. The back magnets have alternating polarity to reduce coupling to external magnetic fields. Attached to the suspension housing are assemblies next to each magnet containing a voice coil, LED, and a photodetector. These coils can be used to apply forces to the optics. The LED/photodector is used as a position sensor, allowing all the low frequency modes to be monitored. Feedback from the position sensors to the coils creates local damping at each mode. Signals from optical levers reflecting off the mirror faces and wavefront sensors are also fed back to these coils. All of these controls were used during the S1 run, but at the design sensitivity only wavefront sensing will be used.

\subsection{Length Sensing and Control}

The entire detector has four degrees of freedom that must be held in place so that all of the optical cavities remain on resonance; the length of each Fabry-Perot arm, the position of the beamsplitter, and the position of the recycling mirror. The beamsplitter is held to control the Michelson interferometer on resonance at a dark fringe on the output port and the recycling mirror controls the resonance in the recycling cavity. These lengths are sensed with an extended version of the PoundDrever-Hall technique ${ }^{10}$ using RF phase modulation sidebands on the carrier laser light. The modulation is applied using Pockels cells and the frequency is chosen so the sidebands are anti-resonant in the Fabry-Perot arms. The carrier light, however,

plenary: submitted to World Scientific on January 16, 2004 


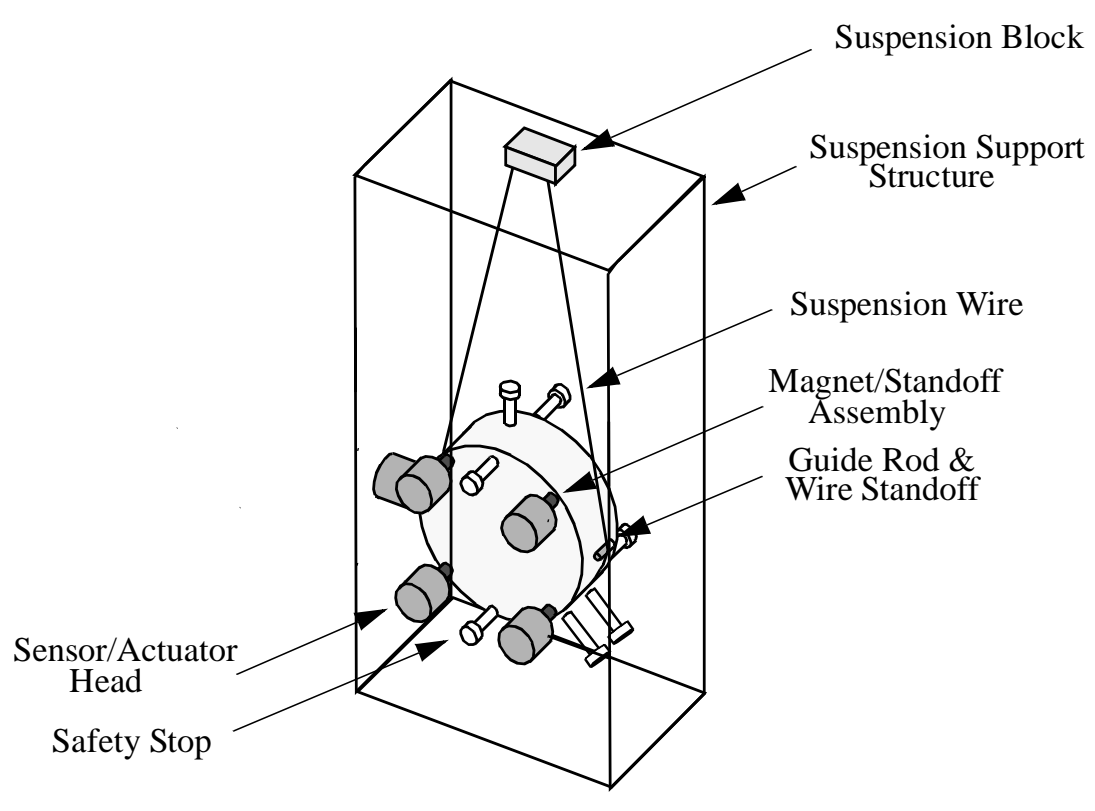

Figure 6. The suspension of a LIGO optic.

is strongly overcoupled in the arms. The recycling cavity is resonant for both the carrier and the sidebands. The sideband light is sent out through the dark port of the Michelson, where it is used as a local oscillator, by introducing a length asymmetry of about $30 \mathrm{~cm}$ between the two beamsplitter-input mirror distances.

Output beams from the interferometer are measured at three locations in the optical plant to determine these four length signals. At the antisymmetric port of the Michelson, the demodulated signal with quadrature phase gives the differential arm length. This is the signal that contains information about gravitational wave events. Light reflected from the recycling mirror demodulated in-phase gives the common mode motion of the arms. This signal is a measure of the laser frequency noise, and is fed back to the laser and mode cleaner to stabilize the laser frequency to the average arm length. Light from inside the recycling cavity, reflected off the antireflection coating of the beamsplitter, gives the remaining degrees of freedom. The in-phase part provides the recycling cavity length and the quadrature phase gives the differential length between the beamsplitter and the input masses. The positions of the photodetectors that measure these signals along with the interferometer layout and degrees of freedom is shown in Fig. 7.

plenary: submitted to World Scientific on January 16, 2004 


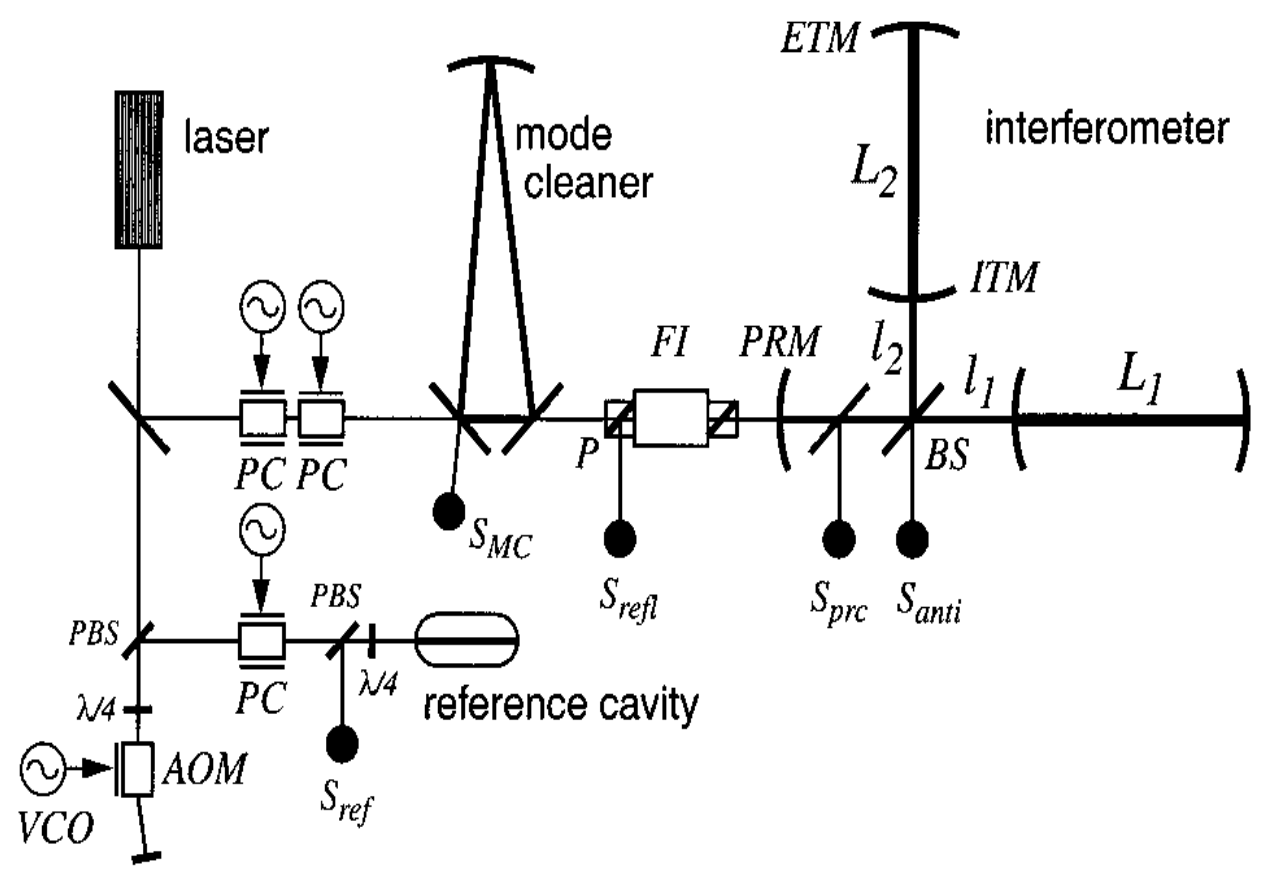

Figure 7. LIGO interferometer showing laser, mode cleaner, the Pockels cells, and photodetectors $\left(\mathrm{S}_{\mathrm{xxx}}\right)$ used to sense the interferometer lengths. The arm cavities have lengths $L_{1}$ and $L_{2}$, the differential arm cavity length is $L_{1}-L_{2}$ and the common mode is $L_{1}+L_{2}$. The Michelson degrees of freedom are the separation of the beamsplitter from the input masses, $l_{1}$ and $l_{2}$. Abbreviations used; PC - Pockels Cell, VCO - Voltage Controlled Oscillator, $\mathrm{S}_{\mathrm{mc}}$ - Mode Cleaner Signal, $\mathrm{S}_{\text {ref }}$

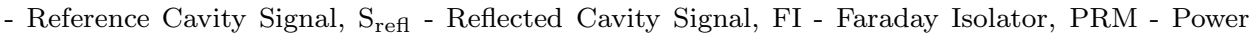
Recycling Mirror, $\mathrm{S}_{\mathrm{prc}}$ - Power Recycling Cavity Signal, $\mathrm{S}_{\text {anti }}$ - Antisymmetric Port Signal, ITM - Input Test Mass, ETM - End Test Mass.

\section{Results from the First LIGO Science Run}

\subsection{Technical Results}

The first science run was intended to collect an amount of real interferometer data to analyze for the presence of gravitational waves. The sensitivity of all interferometers during S1, shown in Fig. 8, was far from the design sensitivity and the run was planned for only 17 days. This made it highly unlikely that a confirmed detection of a gravitational wave would occur during S1, but this data does allow for the setting of upper limits on the event rate and amplitudes of various predicted waves. This upper limit analysis would also provide an opportunity to test the data analysis pipelines with actual data, including non-Gaussian and non-stationary noise. This science data run, similar to the previous engineering runs, also allowed for further evaluation of the interferometers in operation, and to train scientists and operators. The duty cycle of the interferometers was monitored, especially the combined duty cycle of two and three interferometer groupings. The duty cycle results are shown in Table 3.1.

plenary: submitted to World Scientific on January 16, 2004 


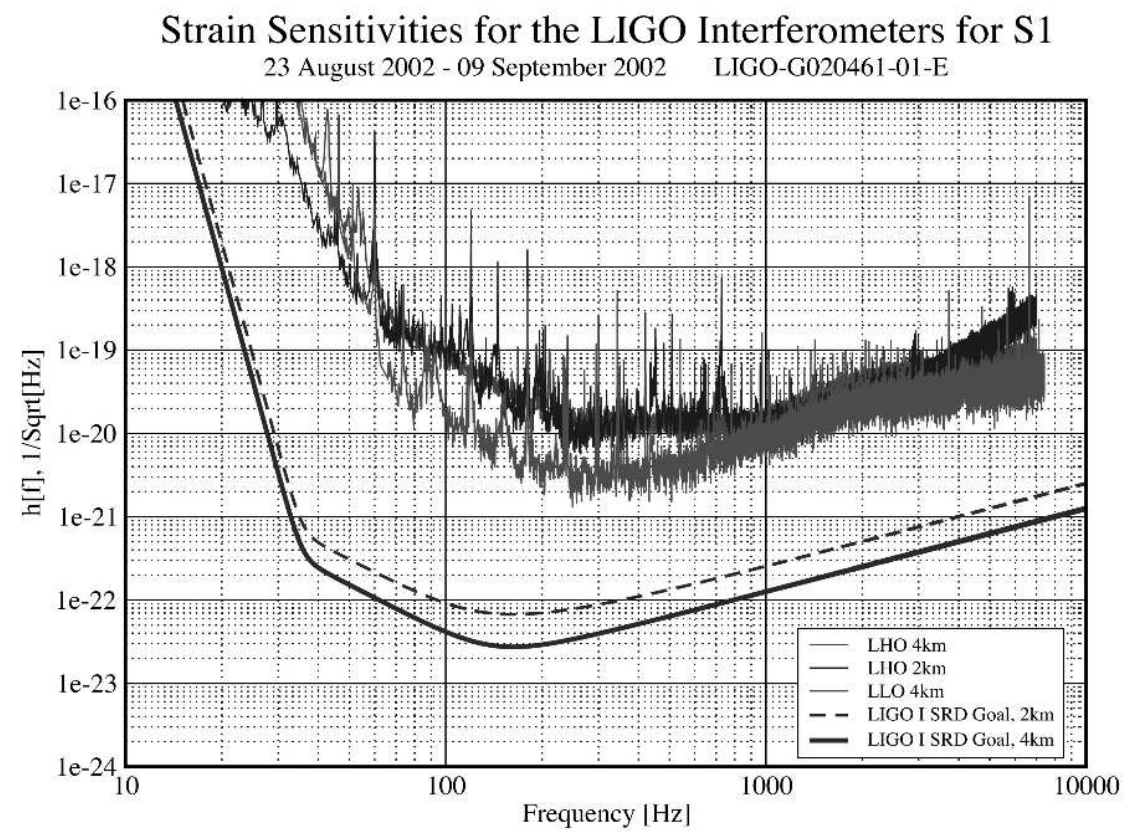

Figure 8. Strain noise in the three LIGO interferometers during S1.

Table 1. Total time locked and duty cycle for LIGO interferometers during S1. The GEO 600 interferometer had a duty cycle of $98 \%$ during S1, so its time in coincidence with LIGO interferometers is constrained by the LIGO duty cycle.

\begin{tabular}{l|c|c}
\hline Detector/combination & Hours of Operation (Coincidence) & Duty Cycle \\
Hanford $4 \mathrm{~km}$ & 235 & $57.6 \%$ \\
Hanford $2 \mathrm{~km}$ & 298 & $73.1 \%$ \\
Livingston $4 \mathrm{~km}$ & 170 & $41.7 \%$ \\
Hanford $4 \mathrm{~km} /$ Hanford $2 \mathrm{~km}$ & 188 & $46.1 \%$ \\
Hanford $4 \mathrm{~km}$ /Livingston $4 \mathrm{~km}$ & 116 & $28.4 \%$ \\
Hanford $2 \mathrm{~km} /$ Livingston $4 \mathrm{~km}$ & 131 & $32.1 \%$ \\
All Three Interferometers & 96 & $23.4 \%$
\end{tabular}

\subsection{Upper Limits on Gravitational Wave Events}

Possible gravitational wave events were divided into four categories for purposes of analysis of S1 data; bursts, inspiralling compact objects, continuous waves, and stochastic background. The data was analyzed using different techniques in order to set upper limits on events in all of these categories. Constraints on time and personpower prevented an exhaustive look at all possible waveforms, so priorities were set based on likelihood of events and maturity of the analysis techniques

plenary: submitted to World Scientific on January 16, 2004 
available.

All analyses (except the periodic sources search) used the LIGO Data Analysis System (LDAS). LDAS provides a distributed software environment with scalable hardware configurations to provide for LIGO's computational needs. The architectural design is based on multiple concurrent data analysis pipelines into which data is fed as it is collected. The data then proceeds down the pipeline where necessary signal analysis procedures are carried out ${ }^{11}$.

\subsection{Burst Events}

Burst events, for purposes of the S1 analysis, are any gravitational wave event of any shape with duration $4 \mathrm{~ms}$ to $100 \mathrm{~ms}$. No other assumptions are made about the waveform of a burst, so matched template analysis can not be used. This category may encompass a large number of astrophysically possible events, but gravitational waves from supernova and gamma ray bursts are of particular interest. A more complete description of the burst analysis can be found in $\operatorname{Ref}^{12}$.

To optimize various parameters used in the burst search, a section of the data, called the "playground data", was set aside and not used for generating the final upper limit. This playground data was composed of about $10 \%$ of the total data set, and was chosen by hand to include as much data variety as possible. To eliminate especially noisy data, the total RMS noise in four frequency bands were monitored and compared to a threshold every six minutes. When band-limited RMS noise exceed the threshold in any band, the six minutes of data was removed from further analysis. To calibrate the data, three sinusoidal excitations were continuously applied to the mirrors during the run. To ensure the calibration of the data was valid, times when these calibration lines gave unreliable results were also rejected from the data analysis stream. Finally, only times when all three LIGO interferometers were providing useful data were included in the analysis. These cuts left 33.5 hours of data to be used in the burst analysis.

This data is then passed to two different event trigger generators; SLOPE ${ }^{13,14}$ and TFCLUSTERS ${ }^{15,16}$. These are used to generate a list of candidate events for each interferometer. SLOPE differentiates the data in time, and compares the slope to a threshold. TFCLUSTERS identifies connected regions in time-frequency space where the power is not consistent with stationary Gaussian noise. After event trigger generation, the analysis pipeline allows for vetoes to be applied based on environmental and interferometer data channels. However, no vetoes were used in the S1 burst analysis. The final step in the pipeline is to generate a list of event candidates using the candidate lists from all three interferometers. Temporal coincidence is required to within the light travel time between the sites $(10 \mathrm{~ms})$ plus the uncertainty in the detection time. For the SLOPE events, a total coincidence window of $50 \mathrm{~ms}$ was used, for TFCLUSTERS $500 \mathrm{~ms}$ was used. For both trigger generators, all triggers that satisfy the triple coincidence that are within $500 \mathrm{~ms}$ of one another are considered the same event. Finally, for TFCLUSTERS events only, a coincidence of $80 \mathrm{~Hz}$ in frequency spacing or less is required. This creates the final list of event candidates.

The list of event candidates will include not only gravitational wave events, but

plenary: submitted to World Scientific on January 16, 2004 
Table 2. Coincident events and confidence bands on the number of excess events in the S1 run from TFCLUSTERS.

\begin{tabular}{l|c}
\hline Coincident Events & 6 \\
Background Events & $10.1 \pm 0.6$ \\
$90 \%$ confidence band & $0-2.3$ \\
$95 \%$ confidence band & $0-3.5$ \\
$99 \%$ confidence band & $0-5.9$
\end{tabular}

some number of random noise events. To determine if the number of candidate events is consistent with only background noise, a background estimate is done. This is done by putting in an artificial time shift in one of the data streams, then performing the same analysis as is done originally. This ensures that gravitational waves will be unable to cause a coincidence, so any events will be solely due to noise. Time shifts between $8 \mathrm{~s}$ (to avoid correlated events) and $100 \mathrm{~s}$ (to minimize dependence on non-stationarity in the noise) were used. It is found that both event trigger generators give varying trigger rates. The results for TFCLUSTERS is shown in Table 3.3. In the presence of the non-stationary noise during S1, SLOPE gave such severe variability that it was not considered suitable for setting an upper limit on gravitational wave events. The problem was due to the use of a single fixed threshold, unlike our implementation of TFCLUSTERS. In future applications of the SLOPE algorithm, we may use an adaptive threshold instead.

Based on the background events in the time shift analysis, the number of coincident events from TFCLUSTERS in Table 3.3 is low. None of these coincident events were seen by SLOPE. The probability of obtaining six or fewer events from TFCLUSTERS based on the measured background is $12 \%$. There is no reason to suspect any systematic error. Alternate methods of estimating the background do not give significantly different background rates.

These results can be used to set an upper limit of the rate of gravitational wave events as a function of amplitude. This is shown in Figure 9. These results assume an isotropic ensemble of waves bathing the Earth with constant amplitude, with all linear polarizations, and a waveform of either Gaussian or sine-Gaussian shape. This model is not motivated by astrophysics, but is meant to be representative of generalized burst events. The amplitude in Figure 9 is shown as the root sum square amplitude spectral density,

$$
h_{r s s} \equiv \sqrt{\int|h(t)|^{2} \mathrm{~d} t} .
$$

Resonant detectors have set more stringent upper limits in the subset of these signals that intersect their frequency band ${ }^{17,18}$. LIGO results will improve both as the interferometer noise is lowered and as longer data sets are accumulated.

\subsection{Inspiralling Binary Neutron Stars}

Compact objects inspiralling together because of gravitational wave emission is one of the best understood astronomical sources of potentially detectable gravitational waves $^{1}$. To create waves in the LIGO band, the inspiralling objects may be any

plenary: submitted to World Scientific on January 16, 2004 


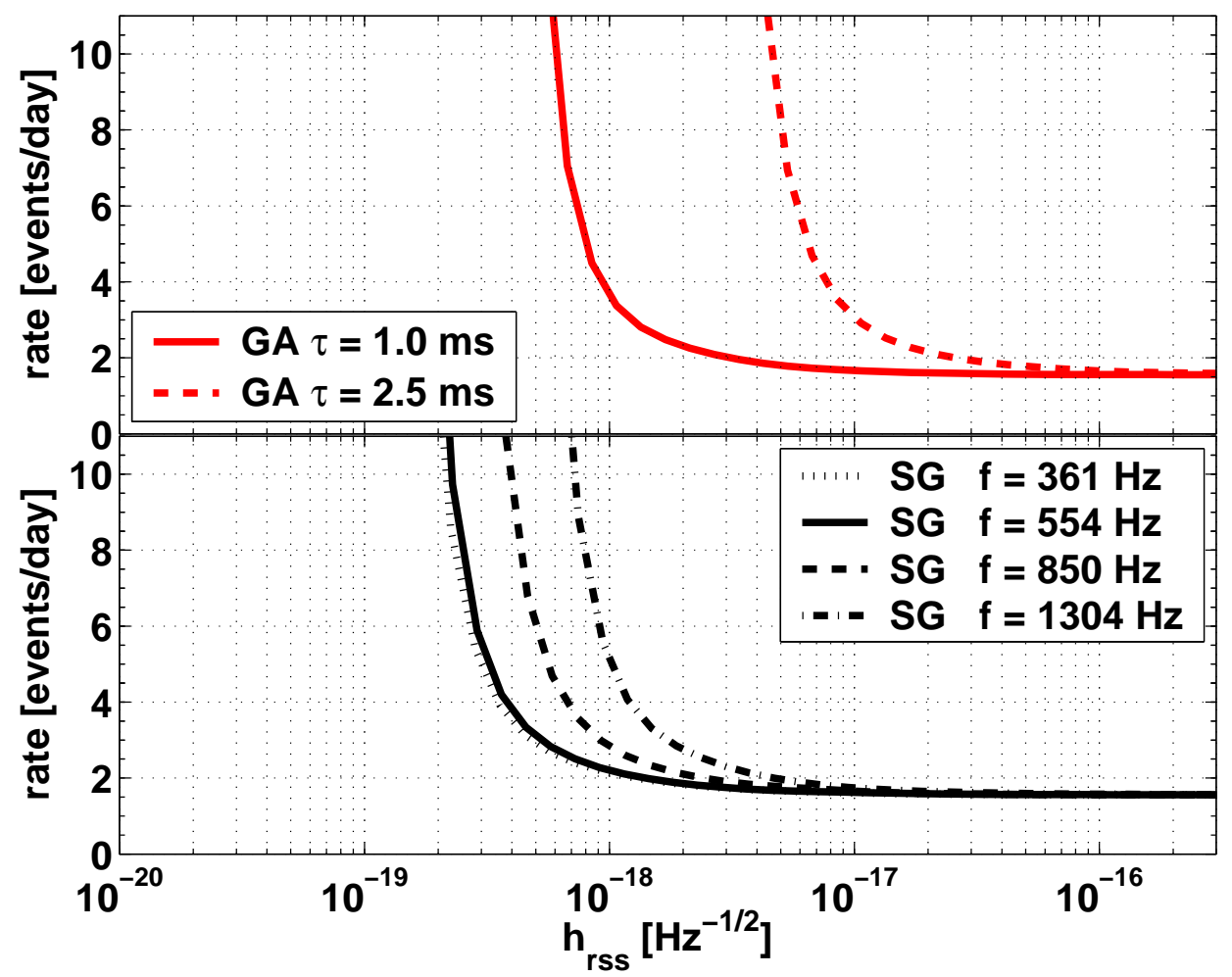

Figure 9. Rate vs. amplitude for detection of specific waveforms using TFCLUSTERS. The top graph shows rates for Gaussian waveforms, with $\tau$ of $1.0 \mathrm{~ms}$ or $2.5 \mathrm{~ms}$. The bottom graph shows rates for sine-Gaussian with central frequencies $361 \mathrm{~Hz}, 554 \mathrm{~Hz}, 850 \mathrm{~Hz}$, and $1304 \mathrm{~Hz}$ and $Q$ of 9 . In each case, the region above and to the right of the curves are excluded at the $90 \%$ confidence level.

combination of solar mass-scale black holes and neutron stars. In the S1 analysis, only binary neutron star waveforms were searched for. This is because of constraints on time and personpower, but also because such systems have been observed to exist $^{19,20,21}$. From the known examples, estimates of the rate of these events can be made ${ }^{22,23}$ which suggest that this source may be detectable by LIGO at the design sensitivity searching for only a few years. A more complete description of the inspiral analysis can be found in $\operatorname{Ref}^{24}$.

The waveform from binary neutron star inspiral can be analytically predicted by using a post-Newtonian expansion ${ }^{25}$, so a template based search can be used. This is the optimal way to search, providing the highest signal-to-noise ratio possible from a given data set. Only data from the LIGO Hanford and Livingston $4 \mathrm{~km}$ interferometers were used in the inspiral analysis.

The templates used were for non-spinning neutron stars, so depend on four parameters; the two neutron star masses $m_{1}$ and $m_{2}$, the arrival phase $\alpha$, and an effective distance $D_{\text {eff }}$. This effective distance depends on the true distance to the

plenary: submitted to World Scientific on January 16, 2004 
Table 3. Event candidates with signal-to-noise ratio (SNR) higher than 8 . The first line shows candidates from when both interferometers were operating, while the last two lines area when only a single interferometer was operating. The first line is for candidates where the Livingston interferometer saw an event with a $D_{\text {eff }}$ less than $51 \mathrm{kpc}$. Coincidence with Hanford was required in this case, and no events survived this coincidence.

\begin{tabular}{l|r|r} 
Interferometers Operating & Number of candidates & Maximum SNR \\
\hline Livingston and Hanford $\left(D_{\text {eff }}<51 \mathrm{kpc}\right)$ & 0 & - \\
Livingston and Hanford $\left(D_{\text {eff }}>51 \mathrm{kpc}\right)$ & 418 & 15.6 \\
Livingston & 786 & 15.9 \\
Hanford & 274 & 12.0
\end{tabular}

source, the location of the source on the sky relative to LIGO's antenna pattern, and the orientation of the orbital plane of the stars. The masses $m_{1}$ and $m_{2}$ were allowed to vary between $1-3 M_{\odot}$. The spacing of the templates in parameter space was chosen so that the most signal-to-noise ratio lost due to template mismatch would be less than 3\%. This led to 2110 different templates being used. Each template, using a frequency dependent weighting due to the noise in the given interferometer, was integrated against the data for all possible arrival times.

Candidate inspirals from either the Hanford or Livingston $4 \mathrm{~km}$ interferometers are found by requiring the signal-to-noise ratio for some template to be higher than 6.5. They must be separated from each other by at least the length of a template. Then they must pass a time-frequency veto designed to reject high amplitude transients. Contributions from eight separate frequency bands were compared to the expected distribution from an inspiral signal ${ }^{26}$. The sum of the squared difference between the actual amount from each frequency and the expected must be less than a threshold. This is known as the $\chi^{2}$ test. Candidates which pass all of these tests are stored as triggers.

Data was not analyzed from either interferometer if the calibration lines were not reliable or if the overall noise in the gravitational wave band was too high. This is similar to conditions used in the burst analysis in Section 3.3. However, unlike the burst search, a veto from an additional data channel was used here. The signal from the common, rather than differential, mode of the arms in the Hanford interferometer was compared to Hanford triggers. This signal is sensitive to frequency noise in the laser. Hanford triggers within one second of events in the common mode signal were vetoed. No instrument vetoes were used with Livingston triggers.

The sensitivity of the Livingston $4 \mathrm{~km}$ interferometer was significantly higher than the $4 \mathrm{~km}$ interferometer at Hanford (see Fig. 8). Because of this, coincidence between triggers from Hanford and Livingston was not required, unless the predicted $D_{\text {eff }}$ from Livingston was less than $51 \mathrm{kpc}$ and hence should be seen in the Hanford data. No coincident triggers were seen in the S1 data. Coincidence would be used to improve confidence in any detection event. The resulting triggers from all of these conditions are shown in Table 3.4.

An upper limit on inspiralling binary neutron star events during S1 was computed from these triggers. The largest signal-to-noise ratio found was used as a threshold. The probability of observing an inspiral signal with signal-to-noise ra-

plenary: submitted to World Scientific on January 16, 2004 
tio $\rho$ greater than this threshold $\rho^{*}$ with some rate $R$ for inspiral events and an observation time $T$ is

$$
P\left(\rho>\rho^{*} ; R\right)=1-e^{-R T \epsilon\left(\rho^{*}\right)},
$$

where $\epsilon(\rho)$ is the efficiency of the analysis for events with signal-to-noise ration $\rho$. The efficiency $\epsilon\left(\rho^{*}\right)$ for $\rho^{*}=15.9$ was determined to be 0.53 from Monte Carlo simulations. A frequentist upper limit with $90 \%$ confidence on $R$ can be found by solving $P\left(\rho>15.9 ; R_{90 \%}\right)=0.9$ for $R_{90 \%}$. With a time $T$ of 236 hours from S1, this gives

$$
R_{90 \%}=170 / \mathrm{y} / \mathrm{MWEG},
$$

where MWEG stands for Milky Way Equivalent Galaxy. Here, a value of the efficiency $\epsilon\left(\rho^{*}\right)$ at the lower end of the 1- $\sigma$ error bars has been assumed to make a conservative estimate. This upper limit is better than previous limits from direct observation $^{27,26}$ by a factor of 26 .

\subsection{Periodic Sources of Gravitational Waves}

Rapidly rotating neutron stars that have some deviation from sphericity will give off gravitational waves. The data from all three LIGO interferometers plus the GEO 600 interferometer were examined for gravitational waves from the fastest rotating pulsar known, J1939+2134. A more complete description of the periodic analysis can be found in $\operatorname{Ref}^{28}$.

The expected signal from a rotating pulsar is an AM and FM modulated sinusoid, Doppler shifted according to the relative motion of the Earth with the pulsar, and amplitude modulated by its motion relative to the antenna pattern of the interferometer. This allows a template based search technique to be used, similar to that used for the inspiralling binary neutron stars. However, the continuous nature of the pulsar signal allows for integration of the signal-to-noise ratio over the complete time that data is available. There are known upper limits to all known pulsars based on their observed spin down rates, as gravitational wave emission cannot be removing energy and angular momentum faster than is seen. The sensitivities of the LIGO and GEO interferometers to periodic sources during S1 along with the spin down upper limits for all known pulsars is shown in Fig. 10.

Two separate data analysis methods were used to set an upper limit on periodic gravitational waves from J1939+2134; a frequency domain technique that yields a frequentist upper limit, and a time domain one that gives a Bayesian upper limit. The frequency domain approach is best for searches over a relatively large parameter space, while the time domain technique is best suited for searches where the phase evolution of the signal is known.

The frequency domain search uses the $F$ statistic method ${ }^{29}$. The logarithmic likelihood function is defined as

$$
\ln (\Lambda)=(s \mid h)-\frac{1}{2}(h \mid h),
$$

where

$$
(s \mid y)=4 \Re \int_{0}^{\infty} \frac{\tilde{s}(f) \tilde{y}^{\star}(f)}{S_{n}(f)} \mathrm{d} f,
$$

plenary: submitted to World Scientific on January 16, 2004 


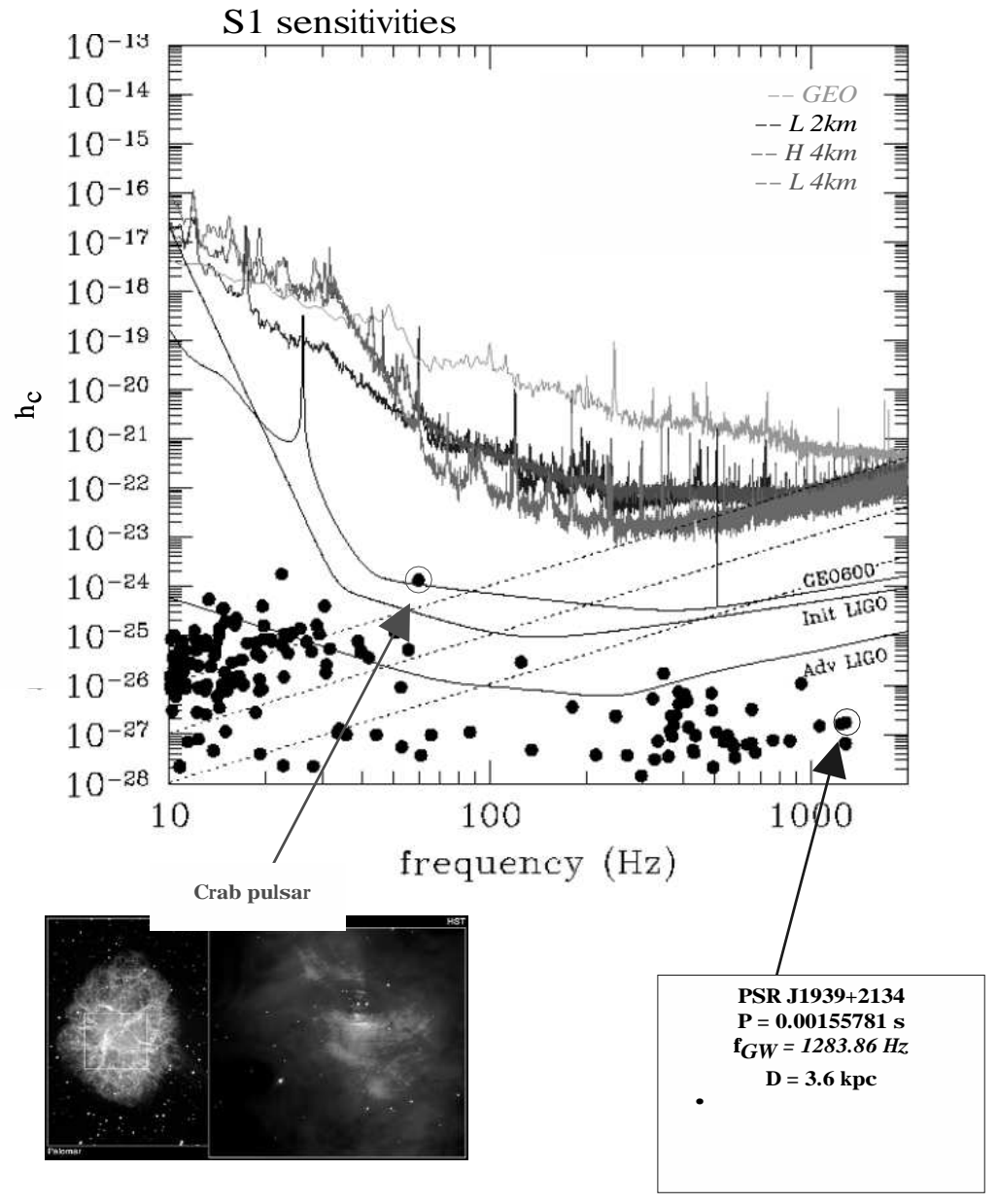

Figure 10. The upper curves show the sensitivities of the four interferometers integrated over their respective up times during S1. The lower curves are sensitivities for a $4 \mathrm{~km}$ LIGO interferometer and GEO 600 with an integration time of one year. The filled circles are upper limits on gravitational wave emission from known radio pulsars based on their observed spin down rates. The circled points are the upper limits for the Crab pulsar and for J1939+2134.

where $s$ is the calibrated detector output time series, $h$ is the template, $\sim$ is the Fourier transform operator, and $S_{n}(f)$ is the one-sided power spectral density of the noise. The $F$ statistic is the maximum value of $\ln (\Lambda)$ with respect to all the unknown signal parameters. For the pulsar J1939+2134, the known parameters are its position on the sky, the frequency $f_{s}$, and the spin down $\dot{f}_{s}$. The unknown parameters are the orientation angle $\imath$, the polarization state of the wave (angle $\psi$ ),

plenary: submitted to World Scientific on January 16, 2004 
Table 4. Upper limits for the gravitational wave amplitude from J1939+2134 at the $95 \%$ confidence level. The time domain search gives a conservative frequentist limit, and the frequency domain search results in a Bayesian limit.

\begin{tabular}{l|c|c} 
Interferometer & Time Domain Search & Frequency Domain Search \\
\hline GEO 600 & $(1.9 \pm 0.1) \times 10^{-21}$ & $(2.2 \pm 0.1) \times 10^{-21}$ \\
Livingston $4 \mathrm{~km}$ & $(2.7 \pm 0.3) \times 10^{-22}$ & $(1.4 \pm 0.1) \times 10^{-22}$ \\
Hanford $4 \mathrm{~km}$ & $(5.4 \pm 0.6) \times 10^{-22}$ & $(3.3 \pm 0.3) \times 10^{-22}$ \\
Hanford $2 \mathrm{~km}$ & $(4.0 \pm 0.5) \times 10^{-22}$ & $(2.4 \pm 0.2) \times 10^{-22}$
\end{tabular}

the initial phase $\phi_{0}$, and the amplitude $h_{0}$. The results shown in the right column of Table 3.5 are the frequentist upper limits that were derived from the $F$ statistic for each interferometer.

The time domain search is better suited to situations where the phase evolution is known. The data to be analyzed for a pulsar signal is heterodyned with a unit amplitude complex function with phase evolution equal to but opposite in sign of the signal. The noise in the interferometer is estimated every minute, and a Bayesian upper limit is calculated. The results are shown in Table 3.5. For both searches, the upper limits are significantly above the limit imposed from the measured spin down rate; $h_{0} \leq 10^{-27}$.

\subsection{Stochastic Gravitational Waves}

There may be a background of gravitational waves analogous to the cosmic microwave background for electromagnetic waves. They are unlikely to be thermally distributed, however. The origin could be cosmological, left over from the big bang or other early universe processes, or it could be astrophysical, an amalgam of more modern sources such as distant supernova or inspirals. See References ${ }^{30,31}$ for more information about stochastic background sources. These sources would appear in LIGO as an otherwise unexplained noise source. Upper limits can be set using a single interferometer, but a much stronger limit comes from cross-correlating multiple detectors. Unlike true instrument noise, the effect of the stochastic background will be correlated in some frequency band. A more complete description of the stochastic background analysis can be found in $\operatorname{Ref}^{32}$.

The spectrum of a stochastic background of gravitational waves is usually described by the dimensionless quantity $\Omega_{\mathrm{gw}}(f)$, the gravitational wave energy density per logarithmic frequency divided by the critical density to close the universe $\rho_{c}$ :

$$
\Omega_{\mathrm{gw}}(f) \equiv \frac{f}{\rho_{c}} \frac{d \rho_{\mathrm{gw}}}{d f}
$$

The one-sided power spectrum of the gravitational wave strain, $S_{g w}(f)$, is then

$$
S_{g w}(f)=\frac{3 H_{0}^{2}}{10 \pi^{2}} f^{-3} \Omega_{\mathrm{gw}}(f),
$$

where $H_{0}$ is the Hubble expansion rate. It is customary to search for a stochastic background for which $\Omega_{\mathrm{gw}}(f)$ is independent of frequency in the LIGO band, as such a spectral behavior is predicted by various cosmological models such as inflation or

plenary: submitted to World Scientific on January 16, 2004 
Table 5. Upper limits for stochastic gravitational waves from pairs of LIGO interferometers. For the value of $\Omega_{0}$ quotes, the Hubble constant was assumed to be $100 \mathrm{~km} /(\mathrm{s} \mathrm{Mpc})$. It scales inversely with $H_{0}$.

\begin{tabular}{c|c|c|c} 
Interferometer pair & $90 \%$ confidence upper limit & Frequency range & Observation time \\
\hline Hanford $4 \mathrm{~km} /$ Livingston $4 \mathrm{~km}$ & $\Omega_{0} \leq 55 \pm 11$ & $40-314 \mathrm{~Hz}$ & $64 \mathrm{hr}$ \\
Hanford $2 \mathrm{~km} /$ Livingston $4 \mathrm{~km}$ & $\Omega_{0} \leq 23 \pm 4.6$ & $40-314 \mathrm{~Hz}$ & $51.25 \mathrm{hr}$
\end{tabular}

cosmic strings. In this case, the gravitatioanl wave strain power spectrum falls as $1 / f^{3}$.

To search for stochastic signals in the S1 data, the background is assumed to be isotropic, unpolarized, stationary, and Gaussian. An incoherent superposition of local sources could cause an anisotropic and/or non-Gaussian background, and may require different data analysis techniques than are used in $\operatorname{Ref}^{32}$. Cross correlation of the output of different detectors provides the best search method, and allows for better limits than just analyzing data from a single interferometer. The cross correlation is done in the frequency domain, and narrow lines from correlated sources, e.g. power lines, are removed. This technique is limited by a frequency dependance set by $c / d$ where $d$ is the distance between the two detectors. This dependance is discussed in detail in $\operatorname{Ref}^{32}$.

The result of this analysis is to set an upper limit on $\Omega_{\mathrm{gw}}(f)$. Arguments from nucleosynthesis already set an upper limit on this value of $10^{-5}$ in the LIGO band. The cross correlation between the two Hanford interferometers actually gives a negative value, which for this reason alone could not be due to a gravitational wave signal. This is due to correlations in the instrument noise, likely from coupling of acoustic noise to the output optics. Useful upper limits can be set using the correlations between Hanford and Livingston, these are shown in Table 3.6. This upper limit is about $10^{4}$ better than the previous broadband limit using interferometric detectors and a factor of about 3 better than the narrowband results from resonant mass detectors.

\section{Advanced LIGO}

Advanced LIGO is the next generation detector currently being researched and designed. The goal is to reach a sensitivity of $200 \mathrm{Mpc}$ for a single $4 \mathrm{~km}$ interferometer, or about $300 \mathrm{Mpc}$ for the three interferometer detector system. At this sensitivity, it is expected that there should be multiple sources a year, if not a week. It will be a complete changeover from the initial LIGO interferometers, with all subsystems replaced, and only the vacuum envelope and other infrastructure retained. A conceptual design for Advanced LIGO was put forth by the LIGO Scientific Collaboration in 1998, and research and development has been ongoing to develop the needed technology. There will be significant improvements in the seismic isolation, suspensions, mirrors, laser, and interferometer configuration. These technologies are discussed below. The expected sensitivity is shown in Fig. 11. In February of 2003, a construction proposal was sent the the US National Science Foundation requesting funds to build Advanced LIGO.

plenary: submitted to World Scientific on January 16, 2004 


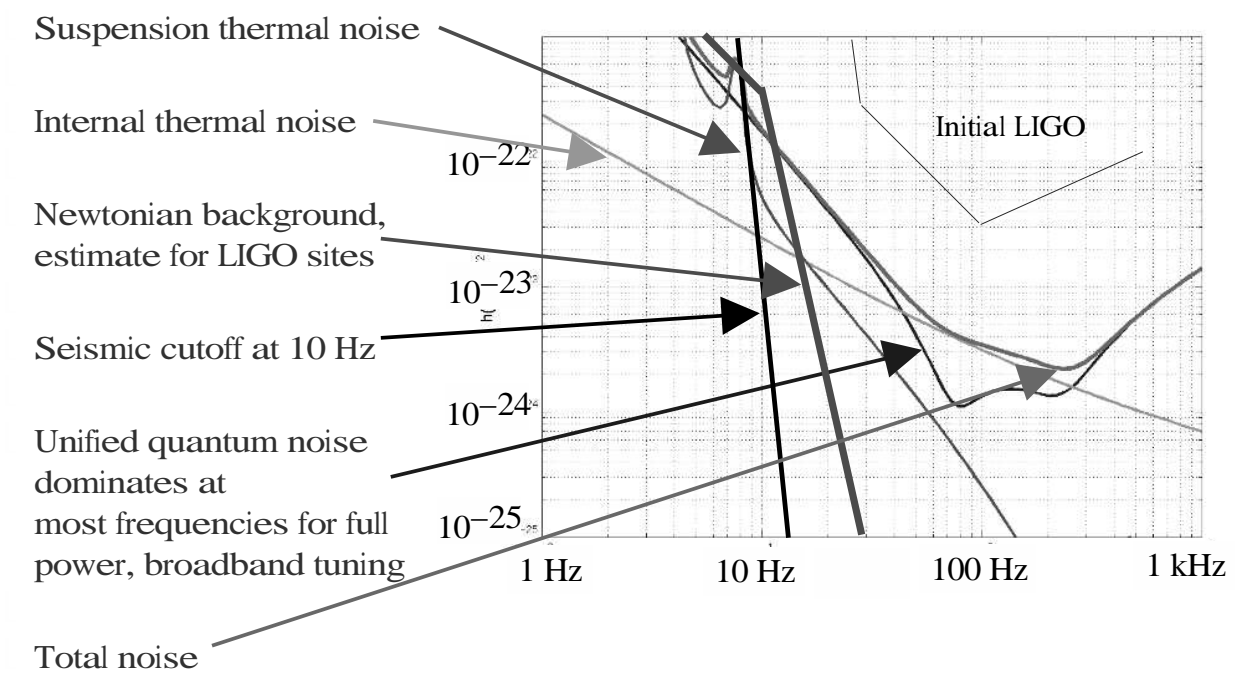

Figure 11. Sensitivity curve for a proposed Advanced LIGO interferometer. The addition of a signal recycling mirror makes radiation pressure and shot noise couple into a single quantum noise. The Newtonian background due to gravitational attraction between the mirrors and the moving ground is expected to dominate over seismic noise at low frequencies. In the middle frequencies, at the most sensitive region, thermal noise from the optical coatings and mirror substrates is the limiting noise source.

\subsection{Seismic Isolation and Suspensions}

The seismic isolation is designed to reduce mirror motion from seismic disturbances to below thermal noise for all frequencies above $10 \mathrm{~Hz}$. This will be done using a two stage, in vacuum, seismic isolation platform with additional low frequency hydraulic pre-isolators external to the vacuum system. The hydraulic pre-isolators are being developed for use on the initial LIGO interferometers to reduce excess seismic noise at Livingston. The in-vacuum stages use a combination of position sensors, seismometers, and geophones to sense motion in the platform. These sensors are then used in feedback loops to apply forces which control all six degrees of freedom $^{34}$.

Below the seismic isolation, the interferometer mirrors hang from suspensions. These are quadruple pendulums, based on triple pendulums used in GEO 600 now $^{35}$. The upper two pendulums use steel wires and blade springs to isolate the mirrors from motion in all directions. The penultimate mass will be made from sapphire or heavy glass and is suspended from above by steel wires. The mirror, which will be

plenary: submitted to World Scientific on January 16, 2004 
sapphire or fused silica, then hangs below the penultimate mass from a fused silica suspension. The use of silica here rather than steel will reduce suspension thermal noise to a level below the quantum noise for most configurations (see Fig. 11). The suspension filaments will be shaped either as ribbons ${ }^{36}$ or dumbbell fibers ${ }^{37}$ to get the maximum dissipation dilution while still maintaining the strength to support the mirrors. The filaments will be welded to silica standoffs which are attached to the masses by hydroxy catalysis bonding ${ }^{38}$ to create low thermal noise but high strength connections.

\subsection{Lasers and Mirrors}

To reduce shot noise, a more powerful laser is needed in Advanced LIGO. Plans are to use a $180 \mathrm{~W}$ injection-locked, end-pumped rod of Nd:YAG which is based on the current GEO 600 laser $^{39}$. This will be able to meet the frequency and intensity noise requirements while greatly increasing the power available. This additional power will mean greater heating in the mirrors and beamsplitter, however. Plans are being developed to use active thermal compensation to reduce thermal lensing in the core optics. This will done using a ring heater, or a scanning $\mathrm{CO}_{2}$ laser. Thermal compensation is also being added to the initial LIGO interferometers to solve thermal lensing problems there.

The interferometer mirrors in Advanced LIGO will be made of sapphire, for its greater ability to handle heat, or fused silica should problems arise with sapphire. The mirrors are planned to be $40 \mathrm{~kg}$ to reduce the effect of radiation pressure from the higher power laser. Single crystal sapphire has been grown this large, and been shown to have acceptable thermal noise characteristics. If the same tantala/silica coatings used in initial LIGO were used for the mirrors in Advanced LIGO, thermal noise from these coatings would place significant limits on the sensitivity. Research is ongoing to understand the cause of thermal noise in optical coatings, to measure it directly, and to develop a coating with lower thermal noise that still meets the optical and thermal requirements ${ }^{8}$.

An additional mirror will be added to the initial LIGO configuration of a powerrecycled Michelson interferometer with Fabry-Perot arm cavities for Advanced LIGO. This is at the output port, called the signal recycling mirror, and it allows the frequency response of the sensitivity curve to be changed and tuned. By changing the position of this mirror by a fraction of the laser wavelength, the maximum sensitivity can be made to occur at different frequencies. By changing the reflectivity, the bandwidth around the central frequency can be adjusted. The quantum noise shown in Fig. 11 shows a signal recycling choice optimized for binary neutron star inspirals, with a maximum sensitivity at about $150 \mathrm{~Hz}$ and a fairly wide bandwidth. A higher frequency, narrow bandwidth choice could be made for gravitational wave pulsar searches, where the particular frequency is known in advance. By lowering the laser power as well as adjusting the signal recycling mirror, Advanced LIGO could be optimized for low frequency performance to enhance searches for stochastic backgrounds, for example. The quantum noise can also be adjusted to best take advantage of the other noise sources, particularly mirror thermal noise, so sensitivity is enhanced in bands where other noise sources are not limiting. Figure 12 shows

plenary: submitted to World Scientific on January 16, 2004 


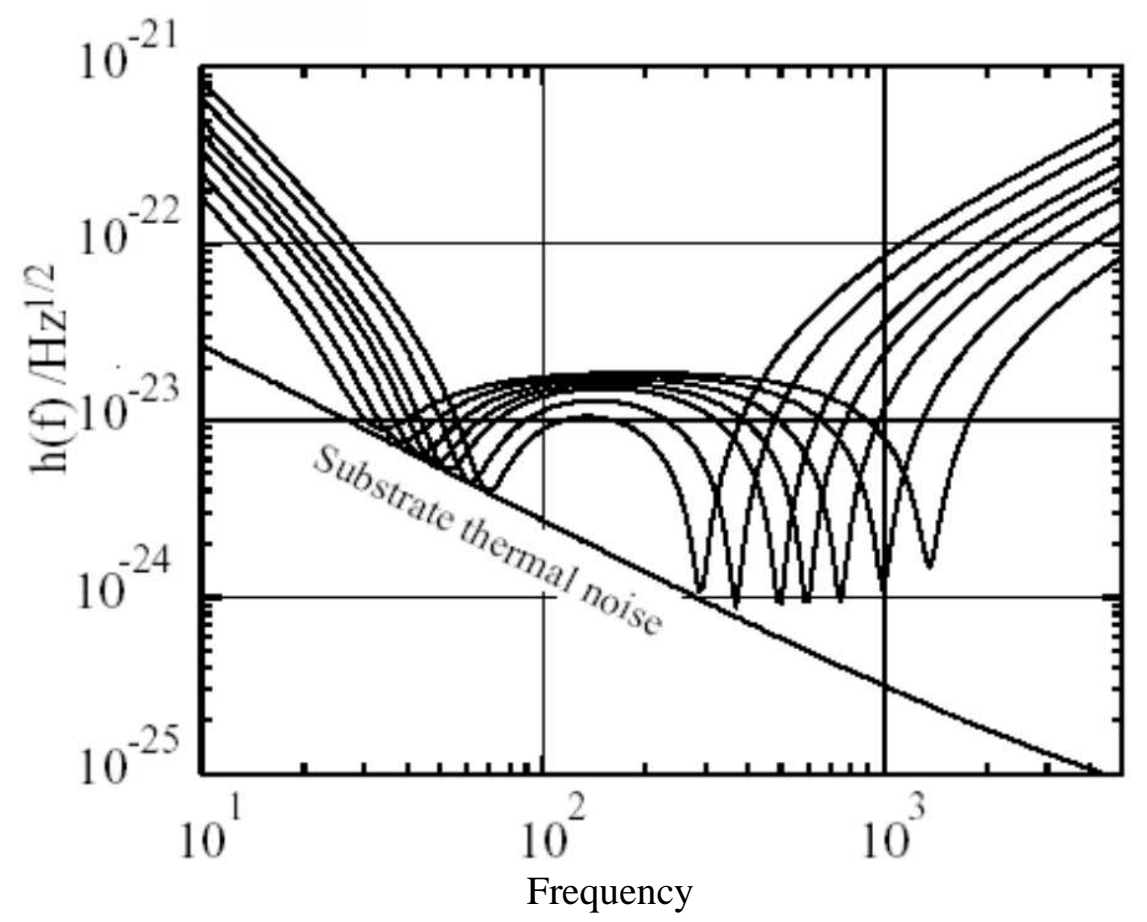

Figure 12. Sensitivity curves for a number of different positions and reflectivities of the signal recycling mirror in Advanced LIGO. These curves are chosen to have high sensitivity in narrow bandwidths, and to avoid the mirror thermal noise that could otherwise limit sensitivity.

a variety of possible narrowband options, as well as the role that mirror thermal noise can play.

\section{Conclusion}

The field of gravitational wave astronomy is rapidly becoming more of a reality, as the LIGO interferometers, in collaboration with GEO 600, are taking data at record sensitivities. The noise in these interferometers is getting close to the design goal, and achieving the target sensitivity is expected to happen soon. The data from the first science run has been analyzed for four categories of gravitational waves, setting upper limits on the rates and amplitudes of them all. Plans are well under way for Advanced LIGO, where every subsystem in the interferometer will be upgraded to take advantage of technological innovations that have occurred since initial LIGO was built. This project was proposed in February of 2003, and should allow for more than a magnitude improvement of sensitivity across a broad band.

plenary: submitted to World Scientific on January 16, 2004 


\section{Acknowledgments}

The LIGO Observatories were constructed by the California Institute of Technology and Massachusetts Institute of Technology with funding from the Nation Science Foundation under cooperative agreement PHY 9210038. The LIGO Laboratory operates under cooperative agreement PHY-0107417. This paper has been assigned LIGO Document Number LIGO-P030058-00-D. The authors gratefully acknowledge the support of the United States National Science Foundation for the construction and operation of the LIGO Laboratory and the Particle Physics and Astronomy Research Council of the United Kingdom, the Max-Planck-Society and the State of Niedersachsen/Germany for support of the construction and operation of the GEO600 detector. The authors also gratefully acknowledge the support of the research by these agencies and by the Australian Research Council, the Natural Sciences and Engineering Research Council of Canada, the Council of Scientific and Industrial Research of India, the Department of Science and Technology of India, the Spanish Ministerio de Ciencia y Tecnologia, the John Simon Guggenheim Foundation, the David and Lucile Packard Foundation, the Research Corporation, and the Alfred P. Sloan Foundation.

\section{References}

1. K. S. Thorne in 300 Years of Gravitation, ed. by S. Hawking and W. Israel (Cambridge, United Kingdom, 1987).

2. B. Abbott et al. (The LIGO Scientific Collaboration), Nuclear Instruments and Methods in Physics Research: A, in press (gr-qc/0308043).

3. B. Wilke et al., Class. Quantum Grav. 19, 1377 (2002).

4. R. Savage, P. King, and S. Steel, Laser Phys. 8, 679 (1998) .

5. S. Whitcomb, G. Billingsley, J Carri, A. Golovitser, D. Jungwirth, W. Kells, H. Yamamoto, B. Bochner, Y. Hefetz, P. Saha, and R. Weiss in Proceedings of the TAMA International Workshop on Gravitational Wave Detection, ed. K. Tsubono, M.-K. Fujimoto, and K. Kuroda (Saitama, Japan, 1996).

6. C. J. Walsh, A. J. Leistner, J. Seckold, B. F. Oreb, and D. I. Farrant, Appl. Optics 38, 2870 (1999).

7. Yu. Levin, Phys. Rev.D 57, 659 (1998).

8. S. D. Penn et al., Class. Quantum Grav. 20, 2917 (2003).

9. J. Giaime, P. Saha, D. Shoemaker, and L. Siever, Rev. Sci. Instrum. 67, 208 (1996).

10. M. W. Regehr, F. J. Raab, and S. E. Whitcomb, Optics Lett. 40, 4988 (2001).

11. S. Anderson, K. Blackburn, A. Lazzarini, W. Majid, T. Prince, and R. Williams in Gravitational Waves and Experimental Gravity: Proceedings of the XXXIVth Recontres de Moriond, ed. J. Tran Thanh Van, J. Dumarchez, S. Reynaud, C. Salomon, S. Thorsett, and J. Y. Vinet (Hanoi, Vietnam, 1999).

12. B. Abbott et al., for submittion to Phys. Rev. D , (gr-qc/0312056).

13. N. Arnaud, F. Cavalier, M. Davier, and P. Hello, Phys. Rev. D 59, 082002 (1999).

14. T. Pradier, N. Arnaud, M. Bizouard, F. Cavalier, M. Davier, and P. Hello,

plenary: submitted to World Scientific on January 16, 2004 
Phys. Rev. D 63, 42002 (2001).

15. J. Sylvestre, Phys. Rev. D 66, 102004 (2002).

16. J. Sylvestre, Ph.D. thesis, Massachusetts Institute of Technology, Cambridge, Massachusetts (2002).

17. Z. Allen et al., Phys. Rev. Lett. 85, 5046 (2000).

18. P. Astone et al., Class. Quantum Grav. 19, 5449 (2002).

19. R. A. Hulse, Rev. Mod. Phys. 66, 699 (1994).

20. J. H. Taylor, Rev. Mod. Phys. 66, 711 (1994).

21. M. Burgay et al., Nature 426, 531 (2003).

22. E. S. Phinney, Astrophys. J. 380, L17 (1991).

23. V. Kalogera et al., in press, Astrphys. J. Lett. , (astro-ph/0312101).

24. B. Abbott et al., submitted to Phys. Rev. D , (gr-qc/0308069).

25. L. Blanchet et al., Phys. Rev. Lett. 74, 3515 (1995).

26. B. Allen et al., Phys. Rev. Lett. 83, 1498 (1999).

27. H. Tagoshi et al., Phys. Rev. D 63, 062001 (2001).

28. B. Abbott et al., in press, Phys. Rev. D , (gr-qc/0308050).

29. P. Jaranowski, A. Królak, and B. Schutz, Phys. Rev. D 58, 063001 (1998).

30. B. Allen in Proceedings of the Les Houches School on Astrophysical Sources of Gravitational Waves, Les Houches, 1995, ed. by J. A. Marck and J. P. Lasota (Cambridge, United Kingdom, 1996).

31. M. Maggiore, Phys. Rep. 331, 283 (2000).

32. B. Abbott et al., submitted to Phys. Rev. D , (gr-qc/0312088).

33. R. R. Caldwell, R. A. Burman, and D. G. Blair, Mon. Not. R. Astron. Soc 324, 1015 (2001).

34. R. Abbott et al., Class. Quantum Grav. 19, 1591 (2002).

35. N. A. Roberson et al., Class Quantum Grav. 19, 4043 (2002).

36. A. Gretarsson et al., Phys. Lett. A 270, 108 (2000).

37. P. Willems, Phys. Lett. A 300, 162 (2002).

38. S. Rowan et al., Phys. Lett. A 246, 471 (1998).

39. I. Zawischa et al., Class. Quantum Grav. 19, 1775 (2002).

plenary: submitted to World Scientific on January 16, 2004 